\title{
Incisional hernia as a late surgical complication of an infertile patient treated for abdominal tuberculosis
}

\author{
Kameshwarachari Pushpalatha $^{1 *}$, Tushar Subhadarshan Mishra ${ }^{2}$, Nerbadyswari Deep ${ }^{3}$
}

\author{
${ }^{1}$ Department of Obstetrics \& Gynaecology, All India Institute of Medical Sciences, Bhubaneswar, Odisha State, India \\ ${ }^{2}$ Department of Surgery, All India Institute of Medical Sciences, Bhubaneswar, Odisha State, India \\ ${ }^{3}$ Department of Radiodiagnosis, All India Institute of Medical Sciences, Bhubaneswar, Odisha State, India
}

Received: 28 July 2015

Revised: 10 September 2015

Accepted: 12 September 2015

\author{
*Correspondence: \\ Dr. K. Pushpalatha, \\ E-mail: pushpak_73@yahoo.com
}

Copyright: ( $)$ the author(s), publisher and licensee Medip Academy. This is an open-access article distributed under the terms of the Creative Commons Attribution Non-Commercial License, which permits unrestricted non-commercial use, distribution, and reproduction in any medium, provided the original work is properly cited.

\begin{abstract}
Abdominal tuberculosis (TB) can be of various forms including peritoneal TB, tuberculous lymphadenopathy, gastrointestinal TB and visceral TB. The potential pathway includes direct spread to the peritoneum from infected adjacent foci, including the fallopian tubes or adnexa, or psoas abscess, secondary to tuberculous spondylitis. The exact stimulus for the inflammatory reaction is not known, but some suggest that it may arise due to a subclinical primary viral peritonitis, as an immunological reaction to gynaecological infections, or due to retrograde menstruation. The diagnosis of extra pulmonary TB can be difficult as it presents with nonspecific clinical and radiological features and requires high degree of suspicion for diagnosis. The abdominal TB, which is not so commonly seen as pulmonary TB, can be a source of significant morbidity and mortality and is usually diagnosed late due to its nonspecific clinical presentation. Approximately $15 \%-25 \%$ of cases with abdominal TB have concomitant pulmonary TB. Hence, it is quite important in identifying these lesions with high index of suspicion especially in endemic areas. Post - operative period in such patients is not smooth. They include prolonged hospital stay, enterocutaneous fistulae requiring re-operation upon failed conservative management, mortality associated with reexploration, late complication being incisional hernia. Here we report a case of large incisional hernia following a laparotomy performed for non-resolving sub-acute intestinal obstruction which turned out to have tubercular origin later. The present case was managed by meshplasty using component separation technique (CST).
\end{abstract}

Keywords: Abdominal tuberculosis, Incisional hernia, Component separation technique

\section{INTRODUCTION}

Abdominal TB was defined as infections of the gastrointestinal tract, peritoneum or intra-abdominal solid organs caused by Mycobacterium tuberculosis. ${ }^{1,2}$ Diagnosis of Abdominal TB was made based on the presenting clinic features and one of the following criteria: (i) positive culture of Mycobacterium tuberculosis from abdominal organ tissue or peritoneal fluid; (ii) positive acid-fast bacilli (AFB) stain from biopsies and (iii) histopathological demonstration of $\mathrm{TB}^{3}$
The prevalence of incisional hernia after laparotomy is reported to be between $11 \%$ and $20 \%$ and incisional hernia recurrence after surgical repair is as high as $45 \%$. $^{4-}$ ${ }^{7}$ Incisional hernias cause pain and other more serious problems, such as bowel obstruction, incarceration, and strangulation. Major abdominal operations have the highest risk for late-onset laparotomy-related complications at 2 and 5 years post- surgery. At 5 years, one fourth of all elderly patients with past history of a major abdominal operation are readmitted for an adhesion-related complication (operative and nonoperative) and $12.0 \%$ undergo repair of an incisional 
hernia. ${ }^{8}$ Here we report a case of large incisional hernia following a laparotomy performed for sub-acute intestinal obstruction of Tubercular origin. The present case was managed by meshplasty using component separation technique (CST).

\section{CASE REPORT}

A 37 year old infertile lady presented to the gynaecology clinic with complaints of weakness and swelling over the abdominal scar along with amenorrhoea of 8 months.

The patient was treated for a non-resolving sub-acute intestinal obstruction by laparotomy 10 years ago. Intraoperative findings comprised of presence of free fluid in the peritoneal cavity, adhesions between the bowel loops. Fluid was sent for Ziehl-Neelsen stain also called as Acid Fast Bacilli staining which demonstrated Mycobacterium Tuberculosis. Subsequently patient was administered Anti-tubercular treatment for 6 months. Within 6 months following the surgery, patient noticed weakness and small swelling over the abdominal scar site on coughing, sneezing etc. As the swelling gradually increased in size with every act of coughing, sneezing, patient sought surgical consultation for which she was offered corrective surgery. She was married for 17 years and apparently consulted gynaecologists for her infertility problem but for no avail. Her previous menstrual cycles were normal till 8 months ago. Her general physical examination revealed nothing abnormal. On abdominal examination, an infraumbilical midline scar was seen. A large hernia of 10x $12 \mathrm{cms}$ seen pronounced on coughing. Upon gynaecologic evaluation, patient was found to have attained premature ovarian failure as evidenced by Serum FSH levels in the postmenopausal range ie,29.36 IU/ml. Contrast Enhanced Computed Tomography of abdomen and pelvis (Figure 1 and 2) showed a large ventral hernia, mild hepatomegaly. Transvaginal ultrasound showed normal sized uterus with an intramural fibroid in the anterior wall measuring 2 × $2 \mathrm{cms}$. Bilateral ovaries were found normal in size and location. Her hemogram and biochemical tests were found within normal range. Chest X-ray and ECG were also normal. As the patient decided to overlook uterine fibroid in the absence of any specific symptoms, only hernia repair was decided upon. Under spinal anaesthesia, laparotomy with meshplasty using Prolene was performed. Postoperative period was uneventful. Patient was discharged with advice on not to strain or act that resulted in increase in the intraabdominal pressure. On subsequent follow-up visits, patient found doing well without any complaints.

\section{DISCUSSION}

Abdominal TB is generally responsive to medical treatment alone, so early diagnosis can prevent unnecessary surgical intervention. In general, with advent of anti-tubercular therapy, surgery is usually reserved for those cases where it is absolutely indicated as in cases of non-resolving intestinal obstruction, perforation and abscess or fistula formation. ${ }^{9}$ It is estimated that one-third of the world population are infected with $\mathrm{TB}^{5}$ and therefore Abdominal TB disease may be caused by reactivation of latent $\mathrm{TB}$. However, re-infection from other family members and following foreign travel should always be considered. It is important to ascertain whether patients were born in a country where TB is endemic. TB often presents within 10 years of migration. Microscopy for AFB of ascitic fluid and pus is often negative (i.e. it has a low sensitivity). Direct visualization and biopsy of the lesion at colonoscopy or laparoscopy/laparotomy yields the best results. ${ }^{3}$
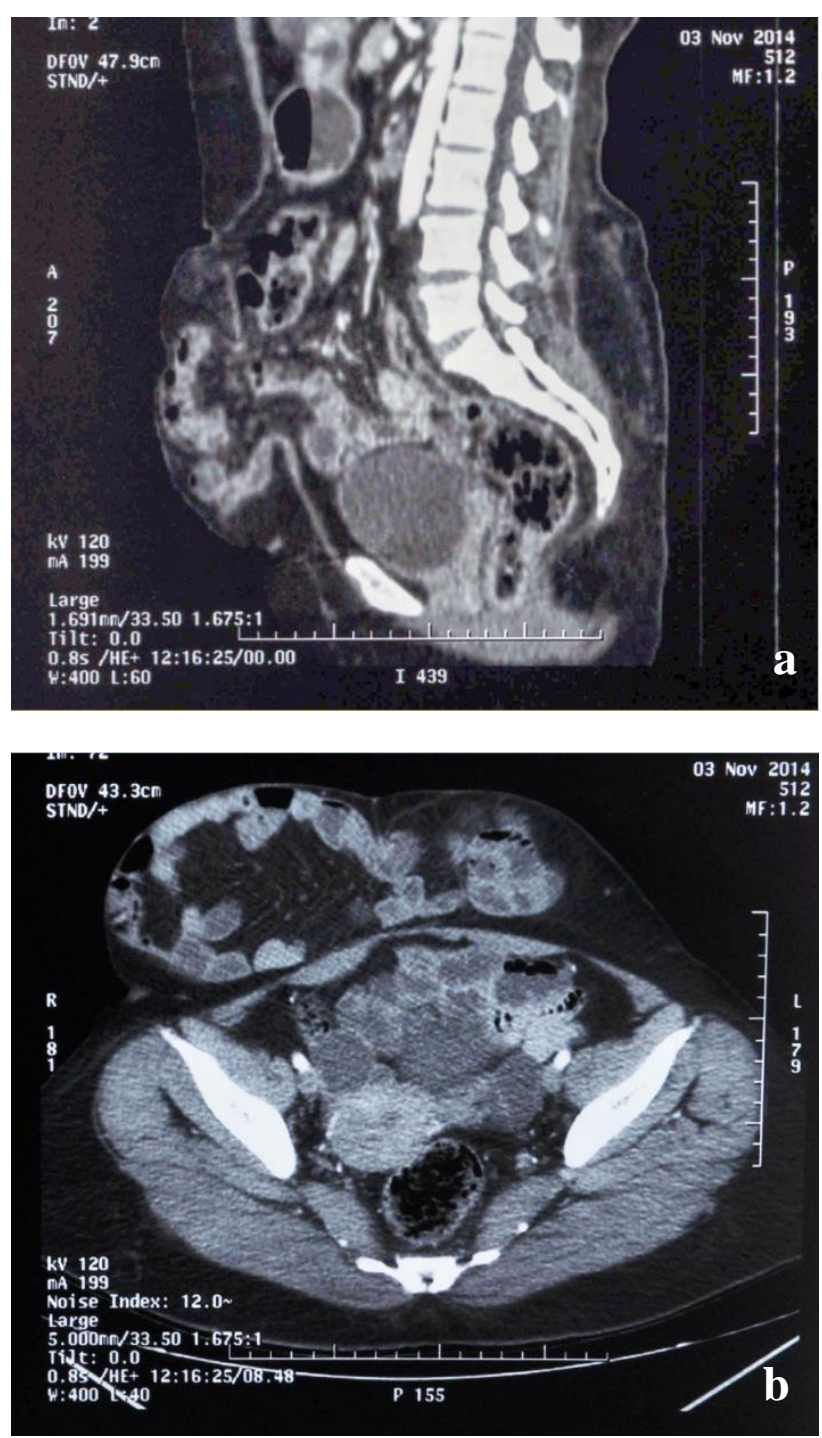

Figure 1 (a \& b): Computed tomography of abdomen showing large ventral defect.

In the study to examine premenopausal risk factors associated with premature ovarian failure (POF) and early menopause (EM) among Korean women, analysis showed that among factors including medical and surgical menopause, lung tuberculosis, hysterectomy, past cancers, and lower number of deliveries before menopause were associated with POF. ${ }^{10}$ Most of the women with this disorder do not have menstrual history, 
specific of POF development, but infertility associated with the diagnosis is the most problematic aspect of the disease. Most experts agree that young women with FOP should replace the hormones the ovary, usually, at least until the age of 50 years. ${ }^{11}$

The management of large ventral defects poses a surgical challenge, particularly since primary repair has reported recurrence rates as high as $50 \% .^{12}$ It is also contraindicated in the presence of gross contamination or infection. Although synthetic mesh carries the risk of mesh infection, exposure, and extrusion, it certainly decreases recurrence rates by up to $20 \% .^{13,14}$

\section{CONCLUSION}

Major abdominal operations carry the highest risk of lateonset complications while laparoscopic operations have the lowest risk of complications. Late-onset laparotomyrelated complications are frequent and their risk extends well beyond the perioperative period. The Component Separation Technique is a safe and effective technique which can be used to treat complex abdominal hernias/defects. Although the technique represents an important method in trunk reconstruction, it promises an acceptable cosmetic outcome with a low rate of hernia recurrence.

Funding: No funding sources Conflict of interest: None declared Ethical approval: Not required

\section{REFERENCES}

1. Lazarus AA, Thilagar B. Abdominal tuberculosis. Dis Mon. 2007;53:32-8.

2. Rasheed S, Zinicola R, Watson D, Bajwa A, McDonald PJ. Intra-abdominal and gastrointestinal tuberculosis. Colorectal Dis. 2007;9:773-83.

3. Mamo JP, Brij SO, Enoch DA. Abdominal tuberculosis: a retrospective review of cases presenting to a UK district hospital. QJM. 2013;106(4):347-54.

4. Mudge M, Hughes LE. Incisional hernia: a 10 year prospective study of incidence and attitudes. $\mathrm{Br} \mathrm{J}$ Surg. 1985;72:70-1.
5. Sugerman HJ, Kellum JM, Reines HD, et al. Greater risk of incisional hernia with morbidly obese than steroid-dependent patients and low recurrence with prefascial polypropylene mesh. Am J Surg. 1996;171:80-4.

6. Anthony $\mathrm{T}$, Bergen PC, Kim LT, et al. Factors affecting recurrence following incisional herniorrhaphy. World J Surg. 2000;24:95-100.

7. Flum DR, Horvath K, Koepsell T. Have outcomes of incisional hernia repair improved with time? A population-based analysis. Ann Surg. 2003;237:12935 .

8. Bensley RP, Schermerhorn ML, Hurks R, Sachs T, Boyd CA, O'Malley AJ, Cotterill P, Landon BE.J Am Coll Surg. Risk of late-onset adhesions and incisional hernia repairs after surgery. 2013;216(6):1159-67

9. Uzunkoy A, Harma M, Harma M. Diagnosis of abdominal tuberculosis: experience from 11 cases and review of the literature. World J Gastroenterol. 2004;10:3647-9.

10. Chang SH, Kim CS, Lee KS, Kim H, Yim SV, Lim YJ, Park SK .Premenopausal factors influencing premature ovarian failure and early menopause. Maturitas. 2007;58(1):19-30.

11. Kalantaridou SN, Nelson LM. Premature ovarian failure is not premature menopause. Ann N Y Acad Sci. 2000;900:393-402.

12. George CD, Ellis $H$. The results of incisional hernia repair: a twelve-year review. Ann R Coll Surg Engl. 1986;68:185-7.

13. Luijendijk RW, Hop WC, van den Tol MP, et al. A comparison of suture repair with mesh repair for incisional hernia. N Engl J Med. 2000;343:392-8.

14. Adekunle S1, Pantelides NM, Hall NR, Praseedom $\mathrm{R}$, Malata CM. Indications and outcomes of the components separation technique in the repair of complex abdominal wall hernias: experience from the cambridge plastic surgery department. Eplasty. 2013;13:e47.

Cite this article as: Pushpalatha K, Mishra TS, Deep $\mathrm{N}$. Incisional hernia as a late surgical complication of an infertile patient treated for abdominal tuberculosis. Int J Reprod Contracept Obstet Gynecol 2015;4:16668. 